\title{
RNAi: an ever-growing puzzle
}

\author{
Ahmet M. Denli and Gregory J. Hannon \\ Cold Spring Harbor Laboratory, Watson School of Biological Sciences, 1 Bungtown Road, Cold Spring Harbor, NY 11724, USA
}

\begin{abstract}
In recent years, sequence-specific gene silencing has been an area of increasing focus, both because of its interesting biology and because of its power as an experimental tool. A growing understanding of one such phenomenon, RNA interference (RNAi), has provided clues that many homology-dependent gene-silencing mechanisms share a common trigger, doublestranded RNA. Recent findings that RNAi and related pathways are involved not only in the response to exogenous pathogenic and endogenous parasitic nucleic acids but also in basic cellular processes, such as gene regulation and heterochromatin formation, have further fueled interest in this rapidly expanding field.
\end{abstract}

The phenomenology of double-stranded RNA (dsRNA)induced silencing has been known for some years in plant systems, as at least one form of CO-SUPPRESSION and as virus-induced gene silencing (VIGS) (reviewed in [1]), before dsRNA itself was known to be the trigger or before similar responses were noted in animals. However, it was the latter observation and the evolution of RNA INTERFERENCE (RNAi; see glossary) as an experimental tool that really drew broad attention to this burgeoning field. In one sense, the story started with a test of the efficacy of antisense RNA in Caenorhabditis elegans. Sense RNA was a logical control but, interestingly, antisense and sense RNAs caused specific silencing of the targeted gene to similar degrees [2]. What might have been considered a failed experiment, in retrospect, built the foundation for coalescence in the field of gene silencing. Fire and colleagues reasoned that the in vitro transcribed RNA preparations used by Guo and Kemphues in their antisense studies were not purely single-stranded RNA and that dsRNA in these preparations might be the key trigger of silencing. Indeed, dsRNA was a much more potent silencing trigger than either strand alone. This phenomenon was named RNA interference (RNAi), distinguishing it mechanistically from classical antisensemediated suppression [3].

\section{The mechanism of RNAi}

An examination of the phenomenology of RNAi in C. elegans and in plants required that any proposed mechanism explain certain unusual characteristics of the process. First, the process had to be flexible and instructed as to its targets by both exogenous and endogenous nucleic acids. These triggers had to serve as a sequence-based template for recognition by machinery that then degraded the target. In some circumstances, mechanisms for signal amplification and for spread of silencing throughout the organism needed to be proposed, although the latter properties are not universal characteristics of RNAi, and will be discussed separately.

In the past several years, the field has come a substantial way towards understanding the mechanism of RNAi through a combination of biochemical and genetic studies in several distinct experimental systems. Many proteins have been linked to RNAi and related phenomena (Table 1 ).

\section{Dicer}

One of the first mysteries to be unraveled, at least to a superficial level of understanding, was how the dsRNAsilencing trigger provided specificity to the silencing machinery. A key breakthrough came from the Baulcombe group. In work on transgene- and virus-induced posttranscriptional gene silencing (PTGS), this group observed the formation of discrete, small RNAs that were complementary to the target of silencing [4]. The discovery of these small RNAs prompted biochemical studies on the activity that formed these RNAs. Could these small RNAs be products of the silencing trigger, and thus possibly key components of the silencing pathway? The answer came when Zamore and colleagues showed that Drosophila embryo extracts could produce small dsRNAs [named SMALL-INTERFERING RNAS (siRNAS)] directly from the dsRNA silencing trigger [5]. In addition, the Hannon group simultaneously found these small RNAs in association with the effector complex of RNAi, RNA-induced silencing complex (RISC; see below), thus implicating them as direct participants in the silencing pathway [6].

RNaseIII family ribonucleases specifically recognize dsRNAs and were thus candidates for the activity that

\footnotetext{
Glossary

Co-suppression: A term that refers to silencing of endogenous loci following the introduction of transgenes. It can also refer to silencing of transgenic loci in a copy-number-dependent fashion.

Dicer: An RNase III family member that produces siRNAs.

PTGS: Post-transcriptional gene silencing, a term used for RNAi in plants where expression is lost without effects on mRNA transcription. RISC: RNA-induced silencing complex. The multicomponent machine that enforces silencing.

RNA interference: RNAi. Becoming an umbrella term to describe homologydependent gene silencing events triggered by double-stranded RNA. Small-interfering RNAs: siRNA. The specificity component of the RNAi machinery.

TGS: Transcriptional gene silencing. As is becoming increasingly clear, dsRNA can also direct changes at the genome level. TGS refers to suppression of transcription in plants following exposure of cells to dsRNAs that are homologous to promoter regions.
} 
Table 1. Proteins and domains involved in RNAi and related phenomena

\begin{tabular}{|lll|}
\hline Protein(s) or protein family & Domains & Domain function \\
\hline Dicer family & RNA helicase & RNA unwinding \\
& PAZ & Putative protein-protein interaction \\
& RNaselll & Ribonuclease \\
dsRNA binding & Putative protein-protein interaction \\
Argonaute family & dsRNA binding & Unknown \\
& PAZ & RNA-dependent RNA polymerization \\
RNA-dependent RNA polymerases & PIWI & RNA unwinding \\
RNA helicases & RdRP & DNA unwinding \\
QDE-3 & Putative RNA helicase & dsRNA binding \\
RDE-4 & DNA helicase & RNA degradation \\
MUT-7 & dsRNA binding & Putative RNA binding \\
Fragile X related protein (dFXR) & Rnase D & Putative RNA binding \\
& KH & Putative RNA binding \\
Vasa intronic gene (VIG) & RGG & Protein-protein interaction \\
SGS3 & RGG & Histone lysine methyltransferase \\
Polycomb proteins & Coiled-coil & Unknown \\
\end{tabular}

produced siRNAs. In Drosophila, Bernstein et al. showed that a class of RNaseIII enzymes produces siRNAs from long dsRNAs in an ATP-dependent manner [7]. These enzymes - named DICERS - are evolutionarily conserved in organisms competent for RNAi, and, in many cases, have been shown to exhibit characteristic biochemical activity. Structural information on archaebacterial RNaseIII [8] has led to a model in which Dicer functions as an anti-parallel dimer to form $\sim 22$ mer siRNAs [9]. In this model, only two of four possible active sites (those on the ends) are competent for cleavage, yielding 22-nucleotide (nt) RNAs rather than the $\sim 11$-nt RNAs that could be produced by similarly arranged bacterial RNase III enzymes. A recent study on recombinant human Dicer shows that dsRNAs are cleaved preferentially at their termini and, in this study, ATP was not required for activity [10]. This raises questions about possible differences between the in vivo dicer complex and recombinant Dicer protein.

When siRNAs from silenced plants are analyzed, two distinct species $(\sim 21 \mathrm{nt}$ and $\sim 25 \mathrm{nt})$ are observed. Recently, it has been suggested that these two kinds of siRNA species are involved in different aspects of silencing. Based on the effects of different viral suppressors of gene silencing and RNAi mutants, the Baulcombe group propose that short siRNAs drive mRNA degradation whereas long siRNAs are involved in systemic silencing and DNA methylation (see below) [11]. Considering the number of Dicer homologs in Arabidopsis, and the differential effects of viral suppressors on these siRNA species, it is plausible that long and short siRNAs might be produced by different Dicer enzymes.

siRNAs are not the only products of Dicer. Dicer mutants in C. elegans have phenotypes that resemble those seen in let-7 and lin-4 mutants, both of which have developmental defects [12-14]. let-7 and lin-4 are noncoding small RNAs [now known generically as microRNAs (miRNAs)] that bind to $3^{\prime}$ untranslated regions (UTRs) of target mRNAs and regulate gene expression at the level of protein synthesis. It is important to note that the complementarity between $3^{\prime}$ UTR sequences and these small RNAs is not perfect. These single-stranded miRNAs are produced by post-transcriptional processing of $\sim 70$ base precursors that form stem-loop structures. Recent data suggest that, in some instances, several clustered miRNAs might be transcribed polycistronically and processed to generate the $\sim 70$ base precursor form in the nucleus [15]. Dicer then processes these $\sim 70 \mathrm{bp}$ hairpins to the mature $\sim 21$-nt form $[12-14,16]$. miRNAs now form a broad class of small RNAs that have been identified in C. elegans, Drosophila, plants and mammals [17-21] The ever-growing number of these developmentally regulated miRNAs suggests an important role for RNAi in endogenous gene regulation.

Dicer proteins appear to be a common denominator in both siRNA and miRNA pathways. However, a key question raised by the discovery of the link between miRNAs and the RNAi machinery, is the degree to which silencing at the level of mRNA degradation might be related to silencing via suppression of protein synthesis. Although no definitive answer has emerged, clues are beginning to emerge from the analysis of RNAi effector complexes.

\section{RNA-induced silencing complex}

At present, the only effector function of RNAi that has been amenable to in vitro analysis is the siRNA-directed degradation of mRNAs. This began with analyses of activities in extracts of Drosophila embryos and cultured $\mathrm{S} 2$ cells, but is now being extended to other organisms, including C. elegans and mammals. To date, four RISC components have been identified in Drosophila and, more recently, four components have been found as part of the mammalian enzyme. However, these do not completely overlap, suggesting either species-, cell type- or developmental stage-specific differences, or the presence of multiple independent effector complexes.

The first protein component to be identified as part of the RISC complex was Argonaute-2, a homolog of C. elegans RDE-1. RDE-1 had previously been shown to be required for RNAi in C. elegans, and belongs to the Argonaute family of proteins [22]. Argonaute proteins are highly basic $\sim 100 \mathrm{kDa}$ proteins that have been linked to RNAi through mutant screens in plants, Neurospora and C. elegans.

Argonaute proteins are comprehensively reviewed in [23]. There are five Argonaute proteins in Drosophila: dAgo1, dAgo2, Piwi, Aubergine and a predicted protein, 
dAgo3. The first four of these proteins have been implicated in RNAi or in potentially related silencing phenomena. Several Argonaute mutants also have developmental defects. Drosophila piwi and aubergine/sting exhibit developmental phenotypes manifested in the gonads, and dAgo1 appears to be essential for viability and normal development of the nervous system. Arabidopsis ago1 and zwille are associated with defects in the shoot apical meristem and exhibit severe phenotypes affecting general plant structure. In C. elegans, two Argonaute homologs, alg-1 and alg-2, exhibit developmental phenotypes in the gonad also. Interestingly, this phenotype is consistent with their role in the metabolism of let-7 and lin-4 miRNAs. How intertwined these developmental and RNAi-related functions are remains to be determined. The answer might lie in further examination of the components of discrete Argonautecontaining RISC complexes, and the identification of their preferential substrates.

Various Argonaute proteins have been isolated in RISC complexes that exhibit a tendency towards either siRNA or miRNA substrates. For example, dAgo1 and dAgo2 are present in separate complexes that preferentially contain siRNAs or miRNAs, respectively [24], and mammalian dAgo1 homolog eIF2C2/hAgo2 resides in a complex associated with miRNAs [21]. Table 2 summarizes the set of component proteins and interactions revealed to date.

The Drosophila homolog (dFXR) of the human fragile X mental retardation protein (FMRP) is another component of the RISC complex. Interestingly, human FMRP has long been known to be a component of ribonucleoprotein complexes, and is believed to regulate a set of genes by affecting protein synthesis. Solidifying the validity of dFXR as a bona fide RISC component, miRNAs, siRNAs and nuclease activity co-immunoprecipitate with dFXR. In addition, transfection of cells with dsRNA against dFXR impairs their RNAi response [24,25]. dAgo2 associates with $\mathrm{dFXR}$ in an RNaseA-resistant manner, suggesting a direct interaction between the two rather than one mediated by mutual binding to target mRNAs [25]. The precise role that dFXR plays in RNAi, and the role that RNAi might play in fragile $\mathrm{X}$ syndrome, remains to be seen.

Recently, purification from mammalian cells yielded a RISC-like complex containing an Argonaute family member and two novel components whose involvement has not yet been demonstrated in other systems. In a study aiming to identify gemin3- [a DEAD-box helicase associated with the survival of motor neurons (SMN) complex] associated

Table 2. Dicer- and Argonaute-associated proteins

\begin{tabular}{|llll|}
\hline $\begin{array}{l}\text { Dicer/Argonaute } \\
\text { family members }\end{array}$ & Organism & Interacting protein(s) & Refs \\
\hline DCR-1 & C. elegans & RDE-4 & {$[53]$} \\
DCR-1 & C. elegans & RDE-1 & {$[53]$} \\
Dicer & Drosophila & dAgo1 & {$[24]$} \\
Dicer & Drosophila & dAgo2 & {$[24]$} \\
Dicer & Drosophila & dFXR & {$[25]$} \\
dAgo2 & Drosophila & dFXR, VIG & {$[24]$} \\
dAgo2 & Drosophila & dFXR, Dmp68 & {$[25]$} \\
elF2C2/hAgo2 & Mammalian & Gemin3, Gemin4 & {$[21]$} \\
elF2C2/hAgo2 & Mammalian & elF2C1/hAgo1 & {$[26]$} \\
\hline
\end{tabular}

proteins, Mourelatos and colleagues identified the human Argonaute member, eIF2C2/hAgo2, as an interacting protein. Using immunoprecipitation and partial purification, they showed that gemin3, eIF2C2/hAgo2, gemin4 (a protein with no known motifs) and miRNAs are present in a $15 \mathrm{~S}$ ribonucleoprotein complex. Like dAgo2, mammalian eIF2C2/hAgo2 is also present in complexes with siRNAs [26], and eIF2C-Gemin complexes are competent for miRNA-directed cleavage of homologous substrates, suggesting that this is a mammalian RISC [27].

In summary, RISC-like complexes are involved in pathways that enforce both siRNA- and miRNA-mediated silencing. Perhaps the most interesting property of these complexes is an apparent flexibility that enables their involvement in these seemingly disparate processes. siRNAs are thought to function through mRNA degradation (Fig. 1). Although miRNAs can function at the level of protein synthesis, a recent finding suggests that miRNAs can also direct mRNA cleavage [20]. One hypothesis is that small RNAs with perfect homology can induce mRNA degradation or mRNA cleavage, and imperfectly paired small RNAs act mainly at the level of protein synthesis. Thus far, the nuclease involved in these complexes remains the most elusive component; however, it is equally unclear as to how miRNA-containing complexes mediate translational suppression.

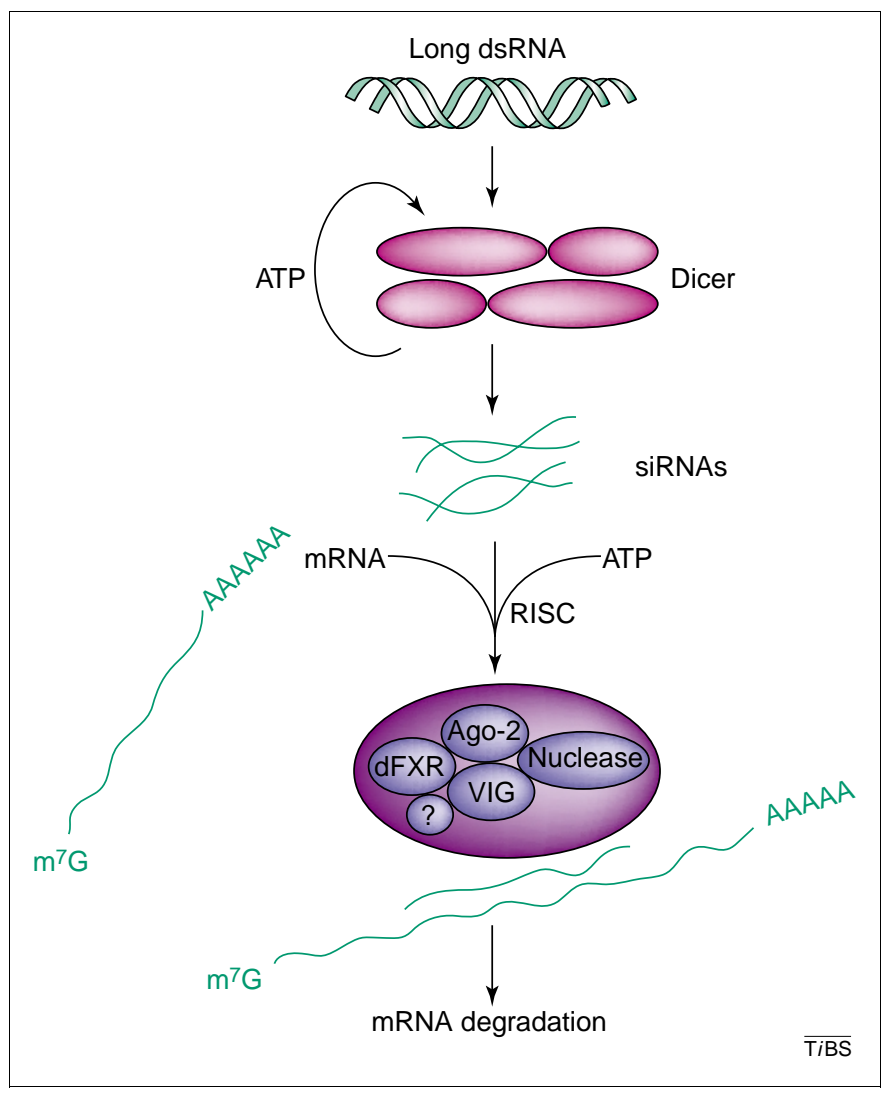

Fig. 1. Present model for the mRNA degradation pathway of RNAi. Anti-paralle Dicer dimers cleave long dsRNAs to form small-interfering RNAs (siRNAs) in an ATP-dependent manner. Recent data from studies on recombinant human Dicer suggest that ATP might be required for release of dsRNA after cleavage, and that mRNAs are cut preferentially at their termini [10]. siRNAs are incorporated in the RNA-induced silencing complex (RISC) and ATP-dependent unwinding of siRNAs activates RISC [56]. Active RISC is thus guided to degrade the specific target mRNAs. 


\section{Amplification, transitive RNAi and systemic silencing}

That very small amounts of dsRNA per cell caused silencing with nearly $100 \%$ penetrance was one of the most striking observations made during early studies of RNAi in C. elegans [3]. This raised the possibility that some aspect of the silencing signal might be amplified. Genetic studies in plants and C. elegans strongly implicate the RNA-dependent RNA polymerases (RdRPs) as key elements of such an amplification loop.

sgs $2 /$ sde 1 mutant plants are defective in the suppression of transgenes [28]. The SGS2 / SDE1 locus encodes a putative RNA-dependent RNA polymerase, which might function either to convert aberrant RNAs into the dsRNA silencing trigger, or to amplify silencing initiated by very small amounts of dsRNA that could originate from the transgene locus. Similarly, in Neurospora, co-suppression is not active in mutants with lesions in its RdRP homolog, qde-1 [29]. By contrast, virus-induced gene silencing is unaffected in sgs2 / sde1 mutants [28]. Viruses encode their own RdRPs that are essential for producing dsRNA during replication, and thus targeting of viral mRNAs might not be dependent on the function of a host RdRP.

Putative RdRPs have also been implicated in conventional RNAi, in which the dsRNA is supplied exogenously, raising the possibility that $R d R P s$ might participate in more than just the generation of the dsRNA silencing trigger. For example, in C. elegans EGO-1 is required for silencing in the germ-line [30], whereas RRF-1 is necessary for silencing in soma [31].

When a restricted portion of a particular mRNA is targeted by RNAi, siRNAs corresponding to other portions of the mRNA can also be detected. This phenomenon is called transitive RNAi and has been observed in C. elegans and plants. In $C$. elegans, these secondary siRNAs are homologous to sequences upstream of those targeted by the primary trigger. This $5^{\prime} \rightarrow 3^{\prime}$ directionality (with respect to the antisense strand) is in agreement with a model where secondary siRNAs are produced following a polymerization event [31]. Similarly, secondary siRNAs can also be observed in plants, but with a twist. In plants, spread is in both the $5^{\prime} \rightarrow 3^{\prime}$ and $3^{\prime} \rightarrow 5^{\prime}$ directions. This spread requires a putative RdRP SGS2/SDE1 and transcription of the transgene [32]. However, the precise mechanisms by which bi-directional spread is achieved remains to be identified.

Evidence for possible involvement of an RdRP activity in Drosophila has also been reported by Lipardi and colleagues. In their model, siRNAs act as primers for secondary dsRNA synthesis, and the majority of RNAimediated degradation is thought to be accomplished by Dicer [33]. However, Zamore's group has shown that replacing $3^{\prime} \mathrm{OH}$ of siRNAs with groups that would not support RNA polymerization has no adverse effect on RNAi [34]. In addition, available (albeit incomplete) human and Drosophila genome sequences lack a recognizable RdRP homolog, and there is a lack of evidence for transitive RNAi in mammals and flies. These observations suggest that trigger amplification and transitive silencing mechanisms might be restricted to only a subset of organisms that are competent for dsRNAmediated silencing.

\section{Natural origins of dsRNA}

The existence of a potent gene silencing mechanism triggered by dsRNA leads immediately to the question of the biological role for such a response. In many organisms, a major source of dsRNA is RNAviruses. RNA viruses form dsRNA intermediates upon replication that might trigger the RNAi response. Several experiments have confirmed a role for RNAi in antiviral defense (reviewed in [35]). In plants, RNAi targets viral mRNAs for degradation [36]. However, many plant viruses have evolved mechanisms to counteract this host response. Viral suppressors of gene silencing (VSGSs) are essential virulence determinants, which become dispensable when RNAi-related pathways in plants are compromised by mutation (reviewed in [37]). It is important to note that VSGSs are not restricted to plant viruses. One RNA virus that infects Drosophila cells - flock house virus (FHV) - also encodes a potential suppressor of silencing that is required for replication. FHV with mutations in b2, a protein that shares genomic organization but not sequence similarity with a plant viral inhibitor of silencing (cucumovirus $2 \mathrm{~B}$ ), can be rescued by suppression of the RNAi pathway in animal cells. Interestingly, FHV b2 also suppresses RNA-induced silencing mechanism in plants, highlighting the conservation of RNAi pathways across kingdoms [38].

What are other targets of RNAi? One emerging theme is that the RNAi machinery provides a conserved mechanism for responding to nucleic acid invaders, in some ways serving as a nucleic acid immune system. Perhaps some of the first indications of this role were experiments from Jorgensen's group, which were designed to overexpress pigmentation genes to enhance flower color in petunia plants. To their surprise, introducing transgenes downstream of a strong promoter caused formation of pigmentfree patches in the transformed plants in which not only transgene, but also the endogenous locus was silenced $[39,40]$. Numerous experiments have suggested that silencing of the pigment gene occurs mainly at the posttranscriptional level. The majority of integrated transgenes form complex arrays. When these transgenic loci are analyzed, a striking percentage contains inverted repeats (IRs) that can form hairpin RNAs upon read-through transcription. Even though IRs appear to be the major inducers of silencing, there are many cases where a singlecopy transgene can also induce silencing (reviewed in [41]). These observations led to the hypothesis that so-called aberrant RNAs form especially during highly active transcription, perhaps owing, in part, to premature termination. There might also be specialized proteins that recognize such RNAs and funnel them into the RNAi pathway.

Besides exogenous nucleic acids, there are numerous endogenous parasitic elements that must be kept largely in check to maintain genome stability. In some cases, control of these mobile genetic elements has been linked to the RNAi machinery. Particularly in C. elegans, some mutants that affect RNAi affect transposon silencing as well. These mutators have increased endogenous transposon activity $[22,42]$. However, the precise mechanism by which RNAi controls the activity of transposons is not yet clear.

One emerging theme is that RNAi responds not only to exogenous nucleic acids but also to endogenous DNA 


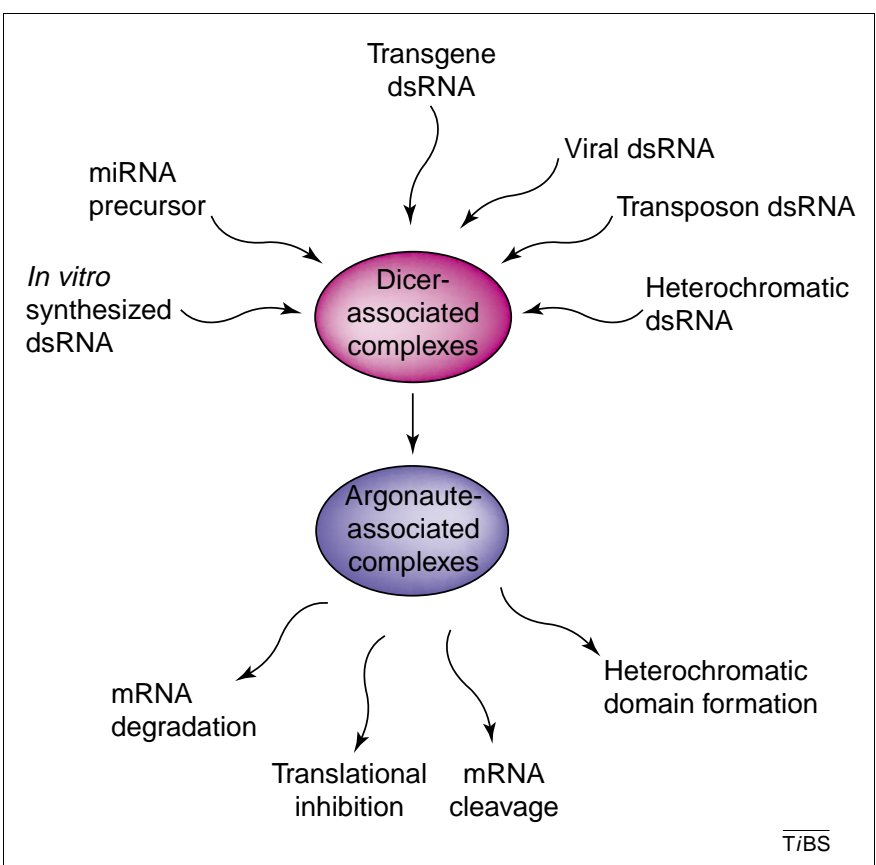

Fig. 2. Multiple inputs to the RNAi machinery can lead to distinct types of silencing events. dsRNA from a variety of sources gets processed by Dicer-associated complexes to guide Argonaute-associated complexes for downstream processes. Dicer and Argonaute family proteins are common denominators in RNA-induced silencing pathways. Genetic studies (reviewed in [57]) suggest that different proteins might be required for silencing by distinct triggers. In addition, biochemical studies suggest correlation of separate Argonaute-associated complexes with different silencing processes (e.g. mRNA degradation versus translational inhibition). One model is that differential interactions of Dicer with trigger-specific proteins trigger the utilization of distinct Argonaute-associated complexes, thus resulting in different outcomes. However, most of the biochemical evidence, to date, suggests that silencing at the level of protein expression and RNA degradation are accomplished by very closely related, if not identical, complexes.

parasites (see Fig. 2). However, recent work has pointed to an even more central role for RNAi in genome maintenance.

\section{dsRNA and heterochromatin}

The initial observation of dsRNA-induced chromatin change was methylation of endogenous sequences that shared homology with viroids in infected plants [43]. This observation was later followed by the finding that dsRNA sharing sequence homology with promoter regions was able to induce gene silencing. In this case, silencing occurred at the transcriptional gene silencing (TGS) level and correlated with de novo methylation of promoter sequences [44]. Unlike the response of PTGS to dsRNA in plants, TGS is heritable [45], and evidence is only now beginning to accumulate suggesting that TGS and PTGS are biochemically related silencing pathways.

Polycomb proteins are chromodomain proteins that transcriptionally repress genes by favoring the formation of closed chromatin structures. These proteins play a role in RNAi in C. elegans and Drosophila. (reviewed in [9]). Furthermore, Piwi is required for co-suppression at both the transcriptional and post-transcriptional levels in Drosophila [46]. These observations established a link between RNAi and chromatin remodeling, and extended the effect of dsRNA on the genome beyond plant systems.

A more direct link was revealed by a recent study in fission yeast. Volpe and colleagues showed that RNAi proteins, namely Dicer, Argonaute and an RNA-directed
RNA polymerase homolog, are required for centromeric silencing [47]. Furthermore, RdRP interacts directly with centromeric repeats [47], and small RNAs complementary to these repeats have been identified [48]. These data strongly implicate the RNAi machinery in the formation of heterochromatin and in the maintenance of genome stability, at least in $S$. pombe [47].

RNAi machinery and repetitive elements in the centromeric region are required for establishment of heterochromatin domains in $S$. pombe, whereas the RNAi machinery is dispensable for its maintenance and inheritance [49]. This is consistent with a model in which heterochromatic domains are formed initially through the RNAi machinery guided by small RNAs. However, once formed, the domains can selfperpetuate through multiple replication cycles.

Tetrahymena is yet another organism in which RNAirelated proteins have been linked both to heterochromatin formation and to the metabolism of repetitive elements in the genome. In Tetrahymena, development of the somatic macronucleus from germ micronucleus involves chromosome rearrangements that lead to elimination of certain sequences. Among these sequences are repetitive elements that show characteristics of transposons. This DNA elimination requires an Argonaute homolog, TWI1, and two chromodomain proteins, PDD1 and PDD3. In addition, a hallmark of heterochromatin, H3K9 methylation, is spatially and temporally limited to the eliminated DNA sequences. Remarkably, targeting of regions for elimination correlates with the presence of small 28-nt RNAs that are homologous to the regions that are lost [50,51].

A central mystery raised by the aforementioned observations is how variants of the RNAi machinery can use small RNA guides to effectively scan either RNA or DNA. Furthermore, how do the effector complexes enforce silencing through changes in RNA metabolism (e.g. mRNA degradation or translational repression) or DNA metabolism (e.g. directing chromatin remodeling)? Answers to these questions might only come as we begin to explore RISCrelated complexes from a more diverse group of model systems.

\section{Concluding remarks}

Through a combination of genetic and biochemical studies, our rough picture of RNAi and related silencing pathways is coming increasingly into better focus. Studies that began with a response to exogenous dsRNAs have provided insight into a surprising variety of cellular phenomena. Despite an expanding list of players identified by both biochemistry and genetics, we still have very little idea of the precise roles played by any of these components, and only the smallest notion of the true breadth of biological functions for these pathways. Nevertheless, one thing seems certain: we will not have to wait long for the next surprising twist in this increasingly complicated plot.

\section{Acknowledgements}

We thank Michelle Carmell and Amy Caudy for critical reading of the manuscript. A.M.D. is a David Koch Fellow of the Watson School of Biological Sciences. G.J.H. is a Rita Allen Foundation Scholar and is supported by an Innovator award from the U.S. Army Breast Cancer Research Program. This work was supported by grants from the National Institutes of Health (GH). 


\section{References}

1 Matzke, M.A. et al. (2002) Homology-dependent gene silencing and host defense in plants. Adv. Genet. 46, 235-275

2 Guo, S. and Kemphues, K.J. (1995) par-1, a gene required for establishing polarity in $C$. elegans embryos, encodes a putative Ser/Thr kinase that is asymmetrically distributed. Cell 81, 611-620

3 Fire, A. et al. (1998) Potent and specific genetic interference by doublestranded RNA in Caenorhabditis elegans. Nature 391, 806-811

4 Hamilton, A.J. and Baulcombe, D.C. (1999) A species of small antisense RNA in posttranscriptional gene silencing in plants. Science 286, 950-952

5 Zamore, P.D. et al. (2000) RNAi: double-stranded RNA directs the ATPdependent cleavage of mRNA at 21 to 23 nucleotide intervals. Cell 101, 25-33

6 Hammond, S.M. et al. (2000) An RNA-directed nuclease mediates posttranscriptional gene silencing in Drosophila cells. Nature 404, 293-296

7 Bernstein, E. et al. (2001) Role for a bidentate ribonuclease in the initiation step of RNA interference. Nature 409, 363-366

8 Blaszczyk, J. et al. (2001) Crystallographic and modeling studies of RNase III suggest a mechanism for double-stranded RNA cleavage. Structure 9, 1225-1236

9 Hannon, G.J. (2002) RNA interference. Nature 418, 244-251

10 Zhang, H. et al. (2002) Human Dicer preferentially cleaves dsRNAs at their termini without a requirement for ATP. EMBO J. 21, 5875-5885

11 Hamilton, A. et al. (2002) Two classes of short interfering RNA in RNA silencing. EMBO J. 21, 4671-4679

12 Grishok, A. et al. (2001) Genes and mechanisms related to RNA interference regulate expression of the small temporal RNAs that control C. elegans developmental timing. Cell 106, 23-34

13 Knight, S.W. and Bass, B.L. (2001) A role for the RNase III enzyme DCR-1 in RNA interference and germ line development in Caenorhabditis elegans. Science 293, 2269-2271

14 Ketting, R.F. et al. (2001) Dicer functions in RNA interference and in synthesis of small RNA involved in developmental timing in C. elegans. Genes Dev. 15, 2654-2659

15 Lee, Y. et al. (2002) MicroRNA maturation: stepwise processing and subcellular localization. EMBO J. 21, 4663-4670

16 Hutvagner, G. et al. (2001) A cellular function for the RNAinterference enzyme Dicer in the maturation of the let-7 small temporal RNA. Science 293, 834-838

17 Lee, R.C. and Ambros, V. (2001) An extensive class of small RNAs in Caenorhabditis elegans. Science 294, 862-864

18 Lau, N.C. et al. (2001) An abundant class of tiny RNAs with probable regulatory roles in Caenorhabditis elegans. Science 294, 858-862

19 Lagos-Quintana, M. et al. (2001) Identification of novel genes coding for small expressed RNAs. Science 294, 853-858

20 Llave, C. et al. (2002) Cleavage of Scarecrow-like mRNA targets directed by a class of Arabidopsis miRNA. Science 297, 2053-2056

21 Mourelatos, Z. et al. (2002) miRNPs: a novel class of ribonucleoproteins containing numerous microRNAs. Genes Dev. 16, 720-728

22 Tabara, H. et al. (1999) The rde-1 gene, RNA interference, and transposon silencing in C. elegans. Cell 99, 123-132

23 Carmell, M.A. et al. (2002) The Argonaute family: tentacles that reach into RNAi, developmental control, stem cell maintenance, and tumorigenesis. Genes Dev. 16, 2733-2742

24 Caudy, A.A. et al. (2002) Fragile X-related protein and VIG associate with the RNA interference machinery. Genes Dev. 16, 2491-2496

25 Ishizuka, A. et al. (2002) A Drosophila fragile X protein interacts with components of RNAi and ribosomal proteins. Genes Dev. 16, 2497-2508

26 Martinez, J. et al. (2002) Single-stranded antisense siRNAs guide target RNA cleavage in RNAi. Cell 110, 563-574

27 Hutvagner, G. and Zamore, P.D. (2002) A microRNA in a multipleturnover RNAi enzyme complex. Science 297, 2056-2060

28 Dalmay, T. et al. (2000) An RNA-dependent RNA polymerase gene in Arabidopsis is required for posttranscriptional gene silencing mediated by a transgene but not by a virus. Cell 101, 543-553

29 Cogoni, C. and Macino, G. (1999) Gene silencing in Neurospora crassa requires a protein homologous to RNA-dependent RNA polymerase. Nature 399, 166-169
30 Smardon, A. et al. (2000) EGO-1 is related to RNA-directed RNA polymerase and functions in germ-line development and RNA interference in C. elegans. Curr. Biol. 10, 169-178

31 Sijen, T. et al. (2001) On the role of RNA amplification in dsRNAtriggered gene silencing. Cell 107, 465-476

32 Vaistij, F.E. et al. (2002) Spreading of RNA targeting and DNA methylation in RNA silencing requires transcription of the target gene and a putative RNA-dependent RNA polymerase. Plant Cell 14, $857-867$

33 Lipardi, C. et al. (2001) RNAi as random degradative PCR: siRNA primers convert mRNA into dsRNAs that are degraded to generate new siRNAs. Cell 107, 297-307

34 Schwarz, D.S. et al. (2002) Evidence that siRNAs function as guides, not primers, in the Drosophila and human RNAi pathways. Mol. Cell $10,537-548$

35 Voinnet, O. (2001) RNA silencing as a plant immune system against viruses. Trends Genet. 17, 449-459

36 Ruiz, M.T. et al. (1998) Initiation and maintenance of virus-induced gene silencing. Plant Cell 10, 937-946

$37 \mathrm{Li}$, W.X. and Ding, S.W. (2001) Viral suppressors of RNA silencing. Curr. Opin. Biotechnol. 12, 150-154

$38 \mathrm{Li}, \mathrm{H}$. et al. (2002) Induction and suppression of RNA silencing by an animal virus. Science 296, 1319-1321

39 Napoli, C. et al. (1990) Introduction of a chimeric chalcone synthase gene into Petunia results in reversible co-Suppression of homologous genes in trans. Plant Cell 2, 279-289

40 van der Krol, A.R. et al. (1990) Inhibition of flower pigmentation by antisense CHS genes: promoter and minimal sequence requirements for the antisense effect. Plant Mol. Biol. 14, 457-466

41 Bernstein, E. et al. (2001) The rest is silence. RNA 7, 1509-1521

42 Ketting, R.F. et al. (1999) Mut-7 of C. elegans, required for transposon silencing and RNA interference, is a homolog of Werner syndrome helicase and RNaseD. Cell 99, 133-141

43 Wassenegger, M. et al. (1994) RNA-directed de novo methylation of genomic sequences in plants. Cell 76, 567-576

44 Mette, M.F. et al. (2000) Transcriptional silencing and promoter methylation triggered by double-stranded RNA. EMBO J. 19 5194-5201

45 Jones, L. et al. (2001) RNA-directed transcriptional gene silencing in plants can be inherited independently of the RNA trigger and requires Met1 for maintenance. Curr. Biol. 11, 747-757

46 Pal-Bhadra, M. et al. (2002) RNAi related mechanisms affect both transcriptional and posttranscriptional transgene silencing in Drosophila. Mol. Cell 9, 315-327

47 Volpe, T.A. et al. (2002) Regulation of heterochromatic silencing and histone H3 lysine-9 methylation by RNAi. Science 297, 1833-1837

48 Reinhart, B.J. and Bartel, D.P. (2002) Small RNAs correspond to centromere heterochromatic repeats. Science 297, 1831

49 Hall, I.M. et al. (2002) Establishment and maintenance of a heterochromatin domain. Science 297, 2232-2237

50 Mochizuki, K. et al. (2002) Analysis of a piwi-related gene implicates small RNAs in genome rearrangement in tetrahymena. Cell 110, 689-699

51 Taverna, S.D. et al. (2002) Methylation of histone h3 at lysine 9 targets programmed DNA elimination in tetrahymena. Cell 110 $701-711$

52 Cogoni, C. and Macino, G. (1999) Posttranscriptional gene silencing in Neurospora by a RecQ DNA helicase. Science 286, 2342-2344

53 Tabara, H. et al. (2002) The dsRNA binding protein RDE-4 interacts with RDE-1, DCR-1, and a DExH-box helicase to direct RNAi in C. elegans. Cell 109, 861-871

54 Mourrain, P. et al. (2000) Arabidopsis SGS2 and SGS3 genes are required for posttranscriptional gene silencing and natural virus resistance. Cell 101, 533-542

55 Dudley, N.R. et al. (2002) Using RNA interference to identify genes required for RNA interference. Proc. Natl. Acad. Sci. U. S. A. 99, 4191-4196

56 Nykanen, A. et al. (2001) ATP requirements and small interfering RNA structure in the RNA interference pathway. Cell 107, 309-321

57 Tijsterman, M. et al. (2002) The genetics of RNA silencing. Annu. Rev. Genet. 36, 489-519 